\title{
In vivo assessment of melanocytic nests in nevi and melanomas by reflectance confocal microscopy
}

\author{
Giovanni Pellacani ${ }^{1}$, Anna Maria Cesinaro ${ }^{2}$ and Stefania Seidenari ${ }^{1}$ \\ ${ }^{1}$ Department of Dermatology, University of Modena and Reggio Emila, Italy and ${ }^{2}$ Department of Pathology, \\ University of Modena and Reggio Emila, Italy
}

\begin{abstract}
In vivo reflectance confocal microscopy is a novel technique for the noninvasive study and diagnosis of the skin. The aim of this study was to describe and characterize the cytological and architectural aspects of cell clusters in melanocytic lesions observed by confocal microscopy, and to correlate them with routine histopathology. A total of 55 melanocytic lesions comprising 20 melanomas, 25 acquired nevi and 10 Spitz nevi were studied by means of reflectance confocal microscopy, dermoscopy and routine histopathology. Three different types of cell clusters at confocal microscopy observation (dense, sparse cell and cerebriform clusters) were identified and correlated with histopathology. Dense clusters appeared characteristic for benign lesions, although present in 13 out of 20 melanomas. Sparse cell clusters were more frequently observable in melanomas, but also sporadically present in one Spitz nevus. Moreover, cerebriform clusters were exclusively observed in five out of $\mathbf{2 0}$ melanomas. Confocal microscopy allowed the in vivo characterization of aspects of melanocytic nests and their exact correlation with histopathology.
\end{abstract}

Modern Pathology (2005) 18, 469-474, advance online publication, 29 October 2004; doi:10.1038/modpathol.3800330

Keywords: histopathology; melanoma; near-infrared confocal microscopy; Spitz nevi

In vivo reflectance-mode confocal scanning laser microscopy (RCSLM) enables the instantaneous visualization of skin structures at a quasihistopathologic resolution, ${ }^{1,2}$ representing a noninvasive approach for the in vivo study of normal skin, ${ }^{1-4}$ inflammatory skin diseases ${ }^{5-8}$ and skin tumours. ${ }^{9-17}$ Some RCSLM features of melanocytic lesions have been recently identified. ${ }^{10-13}$ Studying four melanomas, two lentigo maligna melanomas and 34 melanocytic nevi, distinct cytological features were found at the RCSLM observation. ${ }^{10}$ The presence of cells clustered into nests was reported in melanocytic nevi ${ }^{9,10}$ and in lentigo maligna, ${ }^{13}$ but not described in details.

Differential diagnosis between melanocytic lesions depends on the histopathological analysis of numerous aspects, including the description of cytological and architectural features of melanocytic nests. ${ }^{8}$ Whereas shape and size regularity of the nests, composed by cohesive cuboidal cells, is suggestive of benign lesions, confluent nests with

Correspondence: Professor G Pellacani, MD, Department of Dermatology, University of Modena and Reggio Emilia, Via del Pozzo 71, 41100 Modena, Italy.

E-mail: pellacani.giovanni@unimo.it

Received 30 August 2004; revised and accepted 28 September 2004; published online 29 October 2004 atypical cells, sometimes presenting cellular discohesion, are typical of malignant ones. Moreover, Spitz nevi are characterized by large nests not tending to become confluent, sometimes presenting peculiar artefactual clefts above the nests at the dermal-epidermal junction. ${ }^{19}$ The aim of this study was to describe and characterize the cytological and architectural aspects of cell clusters observed at the RCSLM examination of melanocytic lesions, and to correlate them with histopathology.

\section{Materials and methods}

Subjects

This study included 55 melanocytic lesions comprising 20 melanomas (mean Breslow's thickness $0.81 \mathrm{~mm}), 25$ acquired melanocytic nevi and 10 Spitz nevi. Lesions were selected for the presence of cellular clusters with well-defined borders in the superficial dermis at RCSLM observation from a database of 89 clinically equivocal melanocytic lesions.

Instruments and Acquisition Procedure

RCSLM images were acquired by means of a nearinfrared reflectance confocal laser scanning 
microscope (Vivascope 1000, Lucid Inc, Henrietta, NY, USA). ${ }^{2}$ After acquiring the dermoscopic image, the adapter ring with a diameter of $1 \mathrm{~cm}$ was filled with water and the arm of the RCSLM with the $\times 30$ water immersion objective lens (numerical aperture of 0.9) was placed onto it. High resolution images have a spatial resolution of $0.5-1.0 \mu \mathrm{m}$ in the lateral dimension and $4-5 \mu \mathrm{m}$ in the axial dimension. Each image corresponds to a horizontal section at a selected depth with an effective $475 \times 350 \mu \mathrm{m}$ field of view, with a resolution of $640 \times 480$ pixels and 255 colours. An automated stepper was used to obtain a grid of 16 contiguous horizontal images at a selected depth, constructing a montage image with a $1.9 \times 1.4 \mathrm{~mm}$ field of view ('block' image). A sequence of 30 'block' images was acquired for each lesion at dermal-epidermal junction level and mounted by means of a software developed by us in order to obtain a $7.60 \times 6.65 \mathrm{~mm}$ field of view ('reconstructed image'). ${ }^{20}$

Prior to biopsy, lesion images were recorded employing both digital dermoscopy and RCSLM. In order to have a precise correspondence between RCSLM images and histopathologic section, the RCSLM adapter ring with a $1 \mathrm{~cm}$ diameter was first positioned onto the skin and centred around the lesion. Subsequently, a dermoscopic image of the lesion was acquired by means of a digital videomicroscope (VideoCap 100, DS-Medica, Milan, Italy), ${ }^{21}$ employing a 50-fold magnification and positioning the probe onto the RCSLM adapter ring. After dermoscopic and RCSLM image acquisition, dermoscopic image was resized and rotated for the point by point correspondence with the 'reconstructed' confocal image. ${ }^{22}$ By means of this procedure, high-resolution confocal image could be positioned onto the XY plane of the dermoscopic image for exact pattern correlation. All lesions were then excised, positioning a silk suture at one pole of the specimen to make its orientation easier, and underwent histopathologic examination for diagnostic confirmation and pattern correlation.

\section{Image Description}

For dermoscopic images, traditional dermoscopic features were described for each lesion. ${ }^{23}$

\section{RCSLM features}

On reconstructed RCSLM images, shape and distribution of cellular clusters with well-demarcated borders were described. Cellular clusters were defined as 'few and sparse' when no more than 5 globules per $\mathrm{mm}^{2}$ were present. The term 'focally aggregated' was employed for describing cellular clusters that were present in limited areas of the lesion, whereas when they were numerous and present on the whole examined area, they were described as 'diffusely distributed'. As regards size, small clusters had a major axis inferior to $250 \mu \mathrm{m}$, medium clusters ranged from 250 to $500 \mu \mathrm{m}$, whereas large ones measured over $500 \mu \mathrm{m}$. The cytological characteristics were evaluated on single images with the best resolution $(475 \times 350 \mu \mathrm{m})$, considering cellular size, regularity and distribution. According to their aspect, cellular clusters were divided into three different types. The first one corresponds to compact aggregates of large polygonal cells, with hyporeflecting nuclei and fine granular cytoplasm, forming polyhedral structures (dense clusters) (Figure 1). Roundish nonreflecting structures with a well-demarcated border, containing isolated round to oval cells with dark nucleus and reflecting cytoplasm, sometimes presenting a multilobate aspect were defined as sparse cell clusters (Figure 2). Cellular clusters constituted by confluent aggregates of low reflecting polygonal or elongated structures separated by a low reflecting rim, resulting in a cerebriform appearance, in which cellular nuclei and contour cannot be usually distinguished, were classified as cerebriform clusters (Figure 3).

\section{Histopathologic examination}

On histopathological specimens architectural and cytological features of melanocytic nests were described and correlated to the RCSLM observation.

\section{Results}

At dermoscopy, pigment globules were observed in 17 out 20 melanomas, 23 out of 25 melanocytic nevi and eight out of 10 Spitz nevi. Seven lesions, characterized by darkly pigmented blotches, did not show pigment globules.

On a database of 89 consecutive melanocytic lesions examined at RCSLM for their equivocal appearance, cell clusters were observed in 55 cases $(61.8 \%)$. They were present in 20 out of 32 melanomas $(62.5 \%), 25$ out of 47 melanocytic nevi $(53.2 \%)$ and in 10 out of 14 Spitz nevi $(71.4 \%)$.

RCSLM features of cell clusters as observed by means of RCSLM are reported in Table 1.

\section{Melanomas}

Focally aggregated clusters were observed in nine out of 20 melanomas, whereas in seven melanomas they were diffusely distributed on the examined area and in the remaining four they were few and scattered. Small clusters were observed in 12 lesions, medium-sized clusters in 12 cases and large ones in seven cases, presenting 10 lesions clusters of different size. In 13 melanomas dense clusters, composed by large polygonal cells, were observed (Figure 1c), whereas four lesions 

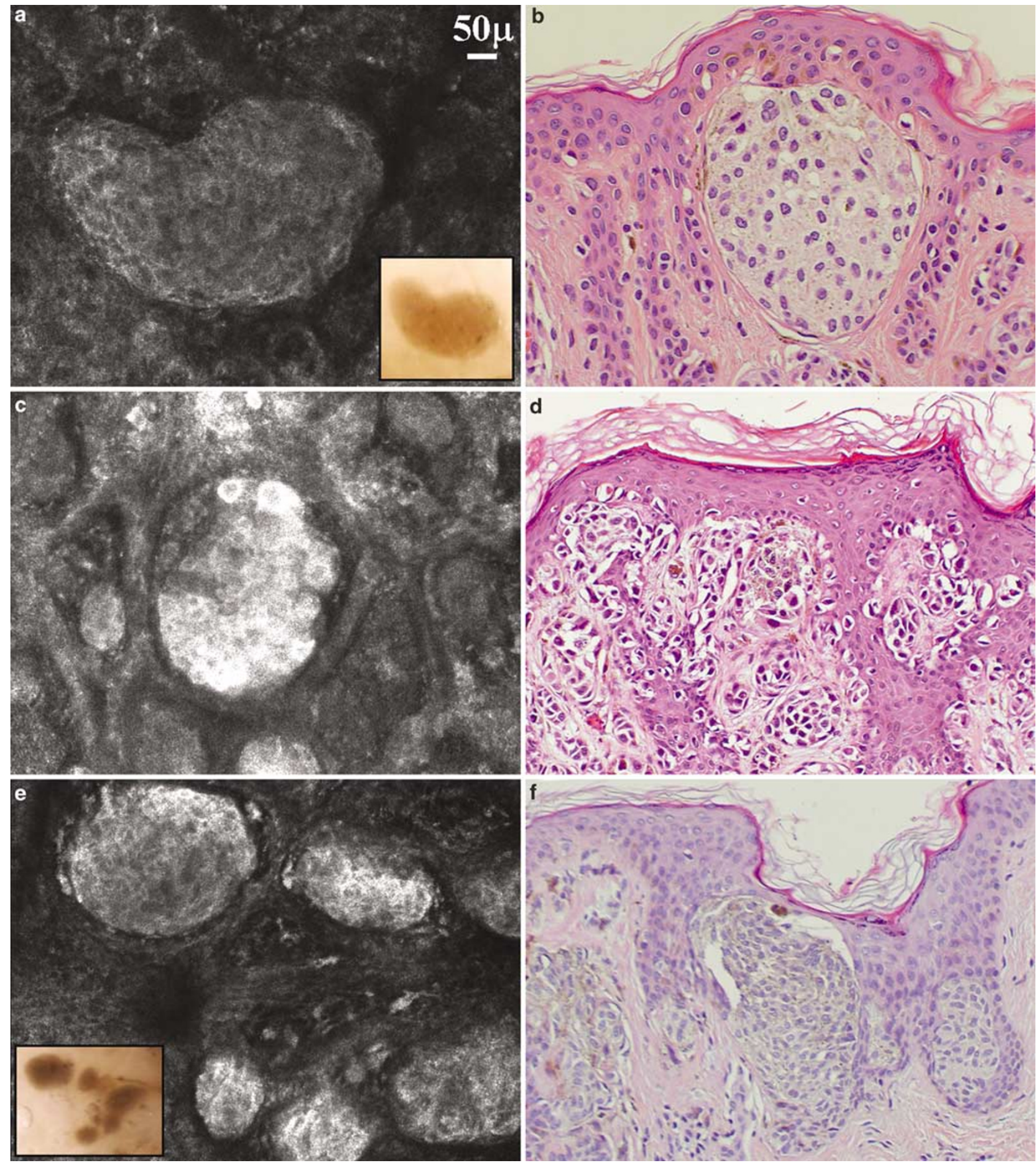

Figure 1 Dense clusters: RCSLM images (a) in an acquired nevus (in the inset: corresponding $\times 200$ dermoscopic image), (c) in a melanoma and (e) in a Spitz nevus (in the inset: corresponding $\times 200$ dermoscopic image) and (b), (d)), (f) their histopathologic correspondence.

had clusters composed by sparse large cells intercalated by thin fibrillar structures giving a multilobate appearance (Figure 2a), and five lesions were characterized by cerebriform clusters (Figure 3a) (in two melanomas two cluster types were contemporary present).

\section{Melanocytic Nevi}

All acquired melanocytic nevi were characterized by dense clusters (Figure 1a). In 14 out of 25 cases, a large number of cell clusters were observable on the whole examined area, forming large conglomerates 
472
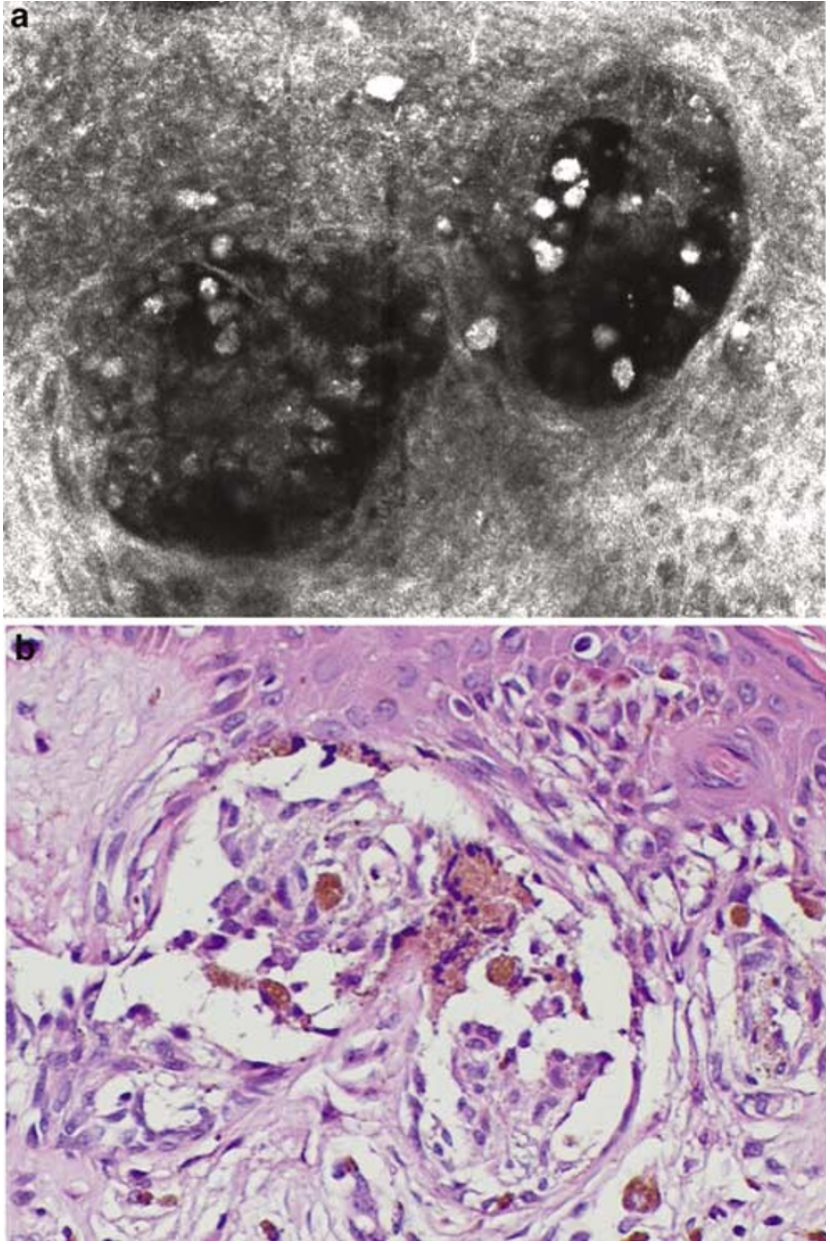

Figure 2 Sparse cell clusters: (a) RCSLM image in a melanoma, presenting a multilobate aspect, and (b) its histopathologic correspondence.

in the centre of three lesions. Five cases showed focal aggregates of cell clusters, whereas few and sparse ones were observable in six cases. All melanocytic nevi presented small and/or mediumsized clusters, associated with large ones in nine out of 25 cases.

\section{Spitz Nevi}

Cellular clusters were diffused on the whole lesion area in three cases, in two of which forming also a well-defined rim at the periphery, whereas six lesions presented aggregates of clusters, in four of which exclusively located at the periphery, and the remaining one was characterized by few and scattered small clusters. All Spitz nevi were characterized by dense clusters (Figure 1e), in one case associated to sparse cell clusters at the periphery.

\section{Histopathologic Examination and Correlation with RCSLM Aspects}

Dense clusters, observed in all benign melanocytic lesions and in 13 out of 20 melanomas,
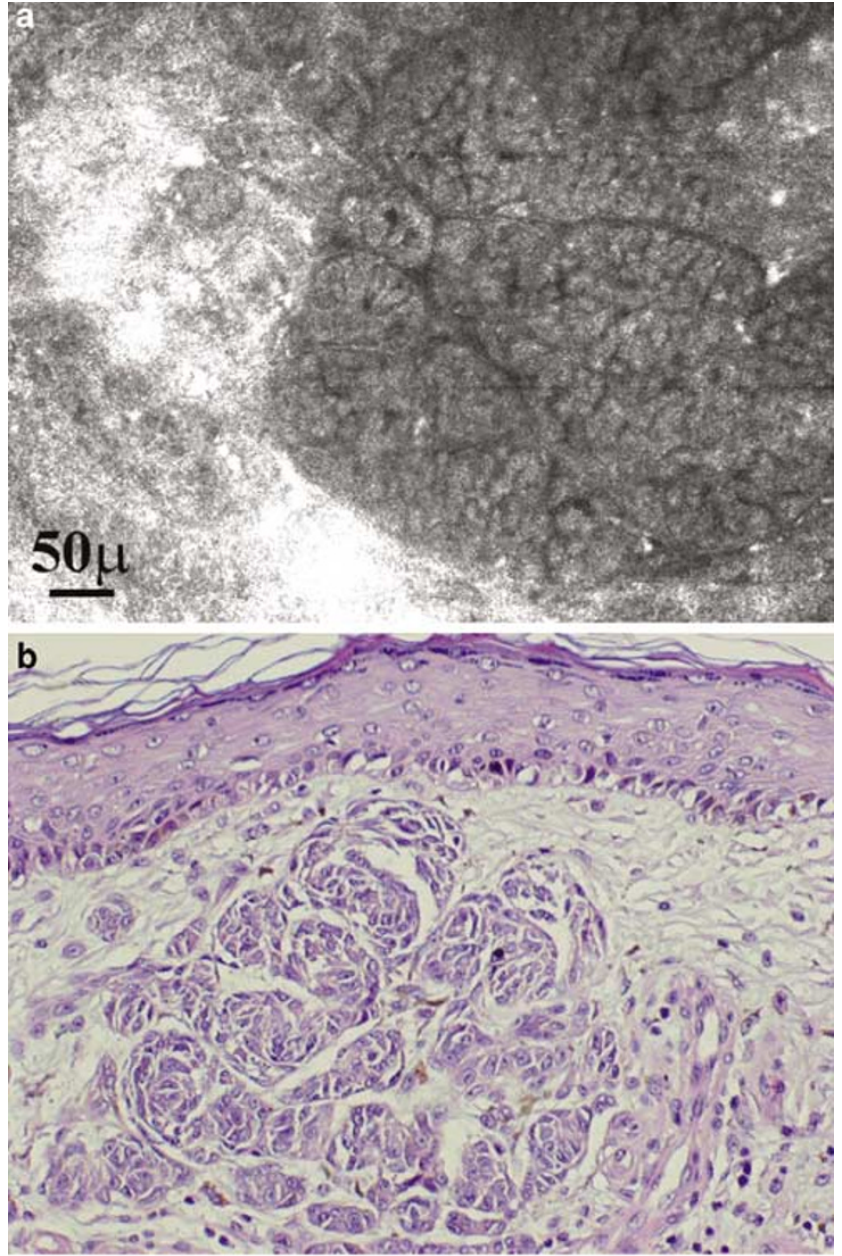

Figure 3 Cerebriform clusters: (a) RCSLM image in a melanoma and (b) its histopathologic correspondence.

Table 1 Features of cellular clusters in melanocytic lesions as observed by RCSLM

\begin{tabular}{lcccc}
\hline & $\begin{array}{c}\text { Melanomas } \\
(20)\end{array}$ & $\begin{array}{c}\text { Melanocytic } \\
\text { nevi (25) }\end{array}$ & $\begin{array}{c}\text { Spitz } \\
\text { nevi (10) }\end{array}$ & Total (55) \\
\hline $\begin{array}{l}\text { Distribution } \\
\text { Few and } \\
\text { sparse }\end{array}$ & $4(20 \%)$ & $6(24 \%)$ & $1(10 \%)$ & $11(20 \%)$ \\
$\begin{array}{l}\text { Focally } \\
\text { aggregated }\end{array}$ & $9(45 \%)$ & $5(20 \%)$ & $6(60 \%)$ & $20(36 \%)$ \\
$\begin{array}{l}\text { Diffusely } \\
\text { distributed }\end{array}$ & $7(35 \%)$ & $14(56 \%)$ & $3(30 \%)$ & $24(44 \%)$ \\
$\begin{array}{l}\text { Size } \\
\text { Small }\end{array}$ & $12(60 \%)$ & $19(76 \%)$ & $7(70 \%)$ & $38(69 \%)$ \\
$\begin{array}{l}\text { (<250 } \mu \mathrm{m}) \\
\begin{array}{l}\text { Medium } \\
(250-500 \mu \mathrm{m})\end{array}\end{array}$ & $12(60 \%)$ & $16(64 \%)$ & $7(70 \%)$ & $35(64 \%)$ \\
$\begin{array}{l}\text { Large } \\
(>500 \mu \mathrm{m})\end{array}$ & $7(35 \%)$ & $9(36 \%)$ & $4(40 \%)$ & $20(36 \%)$ \\
$\begin{array}{l}\text { Type } \\
\text { Dense cluster }\end{array}$ & $13(65 \%)$ & $25(100 \%)$ & $10(100 \%)$ & $47(85 \%)$ \\
$\begin{array}{l}\text { Sparse cell } \\
\text { cluster }\end{array}$ & $4(20 \%)$ & 0 & $1(10 \%)$ & $6(11 \%)$ \\
$\begin{array}{l}\text { Cerebriform } \\
\text { cluster }\end{array}$ & $5(25 \%)$ & 0 & 0 & $5(9 \%)$ \\
\hline & & & & \\
\hline
\end{tabular}


corresponded to well-circumscribed nests composed of large epithelioid cohesive cells with fine dusty melanin, located in the lower epidermis or in the upper dermis. Dense clusters in benign lesions were composed of cohesive cells inside melanocytic nests and clefts were seldom observable in Spitz nevi. Although no differences in dense clusters were observable between benign and malignant lesions by means of RCSLM, compact cellular aggregates with a slight intercellular discohesion were present in melanoma nests at histopathology (Figure 1d). Sparse cell clusters correlated with well-circumscribed nests consisting of unevenly pigmented separated cells. Marked cellular discohesion was characteristic for sparse cell clusters both in melanomas (Figure 2) and in Spitz nevi at histopathology. Corresponding to RCSLM cerebriform clusters, large confluent nests formed by compact malignant cells containing fine dusty melanin were observable in the upper dermis (Figure 3).

\section{Discussion}

RCSLM enables the in vivo study of the skin at a nearly histopathologic resolution, producing pictures of horizontal planes of the epidermis and superficial dermis. Both inflammatory skin disorders and skin tumours were studied by means of this technique, evidencing relevant correspondences with histopathology. Characteristic RCSLM features concerning melanocytes, pigmented keratinocytes and melanophages have been reported. ${ }^{9}$ Moreover, some RCSLM aspects, such as the disarray of the normal honeycombed architecture of the stratum spinosum, the presence of polymorph reflecting cells in the basal cell layers, sometimes spreading upwards in pagetoid fashion, and the presence of dendritic-like structures in superficial layers in lentigo maligna melanomas, appeared to be useful for the distinction between melanocytic nevi and melanomas. ${ }^{10,12,13}$ Although the presence of cell clusters forming nests was previously reported in melanocytic nevi and in lentigo maligna, ${ }^{9,10,13}$ they were not described in melanomas and Spitz nevi. Our study aimed at the characterization of cell clusters in melanocytic lesions, differentiating between different morphological aspects and correlating them with histopathology. The contemporary employment of dermoscopy and RCSLM image reconstruction enabled the exact identification of the dermoscopic pattern and its corresponding confocal feature. ${ }^{24}$ The histopathologic sections drawn along an oriented line enabled a precise correlation with histopathology. Cell clusters by means of RCSLM corresponded to dermoscopic pigment globules in the majority of cases, with the exception of seven lesions where the presence of large dark blotches overshadowed the underlying structures. Dermoscopic globules and RCSLM cell clusters corresponded to histopathologic melanocy- tic nests. According to the morphological presentation, different types of cell clusters were identified by confocal examination. Dense cell clusters consisting of large homogeneous polygonal cells tightly aggregated in a roundish regular structure, were the most frequently observed feature (Figure 1). All benign lesions and 13 melanomas were characterized by dense clusters of various size, sometimes crammed into large aggregates. Although cellular atypia and intercellular discohesion were observable in melanocytic nests of melanomas corresponding to dense clusters at histopathology, no morphological differences between malignant and benign lesions were noticed in dense clusters at RCSLM. Sparse cell clusters appeared as hyporeflecting structures with well-defined borders containing single roundish irregularly scattered cells (Figure 2). These were observed in four out of 20 melanomas and in one out of 10 Spitz nevi, corresponding to a marked intercellular discohesion at histopathology. Thin refractive fibres inside the sparse cell clusters, resulting in a multilobate aspect, were observed only in melanomas, probably attributable to the confluence of malignant cell aggregates. Large multilobate and irregular clusters with a cerebriform appearance were present only in five melanomas and corresponded to confluent nests of malignant cells (Figure 3).

In conclusion, the implementation of a programme for the composition of an overall RCSLM image and the histopathologic section drawn along an established direction, enabled an excellent correlation of the RCSLM aspects with the corresponding dermoscopic pattern and histopathologic features. Moreover, RCSLM enabled the in vivo characterization of different aspects of melanocytic nests in benign and malignant melanocytic lesions. Although the limited number of cases, the identification different types of clusters appeared useful for lesion characterization. In fact, whereas dense clusters were frequently observable both in benign and malignant lesions, sparse cell and cerebriform ones were more frequently present in melanomas compared with nevi. Although RCSLM image acquisition and reconstruction is still time expensive, this tool offers new diagnostic advantages for clinically and dermoscopically difficult to diagnose pigmented skin lesions, allowing the observation of the cytological substrate of dermoscopic structures. Further technical improvement is required to make this technique suitable for routine diagnosis. The combination of dermoscopy and RCSLM may represent a breakthrough in melanoma diagnosis and knowledge on the biology of melanocytic lesions.

\section{References}

1 Rajadhyaksha M, Grossman M, Esterowitz D, et al. In vivo confocal scanning laser microscopy of human skin: melanin provides strong contrast. J Invest Dermatol 1995;104:946-952. 
2 Rajadhyaksha M, Gonzalez S, Zavizlan JM, et al. In vivo confocal scanning laser microscopy of human skin II: advances in instrument and comparison with histology. J Invest Dermatol 1999;113:101-113.

3 Sauermann K, Clemann S, Jaspers S, et al. Age related changes of human skin investigated with histometric measurements by confocal laser scanning microscopy in vivo. Skin Res Technol 2002;8:52-56.

4 Huzaira M, Rius F, Rajadhyaksha M, et al. Topographic variations in normal skin, as viewed by in vivo reflectance confocal microscopy. J Invest Dermatol 2001;116:846-852.

5 Gonzalez S, Rajadhyaksha M, Rubinstein G, et al. Characterization of psoriasis in vivo by reflectance confocal microscopy. J Med 1999;30:337-356.

6 Gonzalez S, Rubinstein G, Mordovtseva V, et al. In vivo abnormal keratinization in Darier-White's disease as viewed by real-time confocal imaging. J Cutan Pathol 1999;26:504-508.

7 Gonzalez S, Gonzalez E, White MW, et al. Allergic contact dermatitis: correlation of in vivo confocal imaging to routine histology. J Am Acad Dermatol 1999;40:708-713.

8 Hicks SP, Swindells KJ, Middelkamp-Hup MA, et al. Confocal histopathology of irritant contact dermatitis in vivo and the impact of skin color (black vs white). J Am Acad Dermatol 2003;48:727-734.

9 Busam KJ, Charles C, Lee G, et al. Morphological features of melanocytes, pigmented keratinocytes, and melanophages by in vivo confocal scanning laser microscopy. Mod Pathol 2001;14:862-868.

10 Langley RGB, Rajadhyaksha M, Dwyer PJ, et al. Confocal scanning laser microscopy of benign and malignant melanocytic skin lesions in vivo. J Am Acad Dermatol 2001;45:365-376.

11 Busam KJ, Hester K, Charles C, et al. Detection of clinically amelanotic malignant melanoma and assessment of its margins by in vivo confocal scanning laser microscopy. Arch Dermatol 2001;137:923-929.

12 Busam KJ, Charles C, Lohmann CM, et al. Detection of intraepidermal malignant melanoma in vivo by confocal scanning laser microscopy. Melanoma Res 2002;12:349-355.

13 Tannous ZS, Mihm MC, Flotte TJ, et al. In vivo examination of lentigo maligna and malignant melanoma in situ, lentigo maligna type by near-infrared reflectance confocal microscopy: comparison of in vivo confocal images with histologic sections. J Am Acad Dermatol 2002;46:260-263.

14 Gonzalez S, Tannous ZS. Real-time, in vivo confocal reflectance microscopy of basal cell carcinoma. J Am Acad Dermatol 2002;47:869-874.

15 Sauermann K, Gambichler T, Wilmert $\mathrm{M}$, et al. Investigation of basal cell carcinoma by confocal laser scanning microscopy in vivo. Skin Res Technol 2002;8:141-147.

16 Goldgeier M, Alessi Fox C, Zavislan JM, et al. Noninvasive imaging, treatment, and microscopic confirmation of clearance of basal cell carcinoma. Dermatol Surg 2003;29:205-210.

17 Tannous Z, Torres A, Gonzalez S. In vivo real-time confocal reflectance microscopy: a noninvasive guide for Mohs micrographic surgery facilitated by aluminum chloride, an excellent contrast enhancer. Dermatol Surg 2003;29:839-846.

18 Elder D, Elenitsas R. Benign pigmented lesions and malignant melanoma In: Elder D, Elenitsas R, Jaworsky C, Johnson B (eds). Lever's Histopathology of the Skin, 8th edn. Lippincott-Raven: Philadelphia, PA, 1997, pp 625-684.

19 Ackerman AB, Magana-Garcia M. Naming acquired melanocytic nevi. Am J Dermatopathol 1990;12:193-209.

20 Pellacani G, Cesinaro AM, Grana C, et al. In vivo confocal scanning laser microscopy of pigmented Spitz nevi. Comparison of in vivo confocal images with epiluminescence microscopy and routine histology. J Am Acad Dermatol 2004;51:371-376.

21 Seidenari S, Burroni M, Dell'Eva G, et al. Computerized evaluation of pigmented skin lesion images recorded by a videomicroscope: comparison between polarizing mode observation and oil/slide mode observation. Skin Res Technol 1995;1:187-191.

22 Pellacani G, Cesinaro AM, Longo C, et al. Microscopic in vivo description of cellular architecture of dermoscopic pigment network in nevi and melanomas. Arch Dermatol, in press.

23 Argenziano G, Soyer HP, Chimenti S, et al. Dermoscopy of pigmented skin lesions: results of a consensus meeting via the Internet. J Am Acad Dermatol 2003; 48:679-693.

24 Pellacani G, Cesinaro AM, Seidenari S. In vivo confocal reflectance microscopy for the characterization of melanocytic nests and correlation with dermoscopy and histology. Br J Dermatol, in press. 